Revue d'histoire de l'enfance « irrégulière »

Le Temps de l'histoire

2| 1999

Cent ans de répressions des violences à enfants

\title{
La loi du 19 avril 1898 et les institutions
}

Éric Pierre

\section{(2) OpenEdition}

Journals

Édition électronique

URL : http://journals.openedition.org/rhei/45

DOI : $10.4000 /$ rhei. 45

ISBN : 978-2-7535-1639-7

ISSN : $1777-540 \mathrm{X}$

Éditeur

Presses universitaires de Rennes

Édition imprimée

Date de publication : 15 novembre 1999

Pagination : 113-127

ISSN : 1287-2431

Référence électronique

Éric Pierre, «La loi du 19 avril 1898 et les institutions », Revue d'histoire de l'enfance « irrégulière » [En ligne], 2 | 1999, mis en ligne le 30 juillet 2010, consulté le 03 décembre 2020. URL : http:// journals.openedition.org/rhei/45 ; DOI : https://doi.org/10.4000/rhei.45

(c) PUR 


\section{La loi du 19 avril 1898 et les institutions}

La justice des mineurs connaît au tournant du XXe siècle une remarquable effervescence. Elle "intéresse les esprits" et "passionne les cœurs". ${ }^{(2)}$ Jamais, depuis les débats pénitentiaires des années $1830-1840,{ }^{(3)}$ les spécialistes du sujet n'ont autant débattu des questions concernant les jeunes délinquants, les enfants assistés, ou encore les mineurs en danger. Quatre instances au moins, aux fonctions et aux statuts juridiques divers, réunissent ces spécialistes. ${ }^{(4)}$ Leurs séances, studieuses et souvent passionnées, donnent lieu à la présentation d'études sur la situation des mineurs enfermés, à l'exposition de programmes de réformes des établissements, à l'élaboration de projets législatifs qui seront proposés aux parlementaires. Les congrès nationaux et internationaux, qu'ils soient pénitentiaires ou de protection de l'enfance, permettent de nouveaux échanges. ${ }^{(5)}$ Fonctionnaires, administrateurs, philanthropes, ${ }^{\left({ }^{(}\right)}$juristes et praticiens du droit y confrontent des modèles qu'ils souhaitent voir diffuser largement. (")

En France, depuis le début des années 1870, les réflexions s'orientent dans de nombreuses directions. Il s'agit tout à la fois d'améliorer le fonctionnement des établissements existants ou d'en créer de nouveaux, mieux adaptés à leur fonction éducative, de réformer la correction paternelle, ${ }^{(8)}$ de dépénaliser le traitement de certains mineurs délinquants (en particulier les plus jeunes), enfin de punir les véritables responsables de la déchéance des jeunes : les parents incapables ou indignes, ceux qui maltraitent ou négligent l'éducation de leurs enfants. La loi d'avril 1898, qui vient compléter et améliorer celle de juillet 1889 sur les enfants moralement abandonnés, ${ }^{(9)}$ intervient à plusieurs de ces niveaux, surtout depuis que le sénateur Bérenger, profitant des débats parlementaires, a accolé une réforme de l'article 66 du code pénal sur les jeunes détenus aux disposi-

\section{Eric Pierre $^{(1)}$}

(1) Maître de conférences, Université

d'Angers.

(2) Expressions empruntées à une thèse de droit : Albert GALLOIS, La Protection de l'enfance maltraitée. Le châtiment du coupable. Les mesures protectrices de la victime

(Etude critique de la loi du 19 avril 1898), Paris, Delmar, 1899, 248 p.

(3) Sur les débats pénitentiaires des années 1830-1840, Jacques-Guy PETIT, Ces peines obscures. La prison pénale en France 1780-1875, Paris, Fayard, 1990, pp. 219260. Sur la question des jeunes détenus à la même période, Eric PIERRE, "Débats pénitentiaires, politiques correctionnelles et vote de 
la loi de 1850", in

Michel CHAUVIERE,

Pierre LENOËL, Eric

PIERRE, Protéger l'enfant.

Raison juridique et pra-

tiques socio-judiciaires

(XIXe-XXe siècles),

Rennes, Presses Univer-

sitaires de Rennes, 1996,

pp. 71-105.

(4) Il s'agit de la

Société générale des prisons, du Comité de défense des enfants traduits en justice de Paris, de l'Union des sociétés de patronage et du Conseil supérieur des prisons. Sur ces réseaux réformateurs : Martine KALUSYNSKI, "Réformer la Société, les hommes de la Société générale des prisons, 1877-1900", Genèses, $\mathrm{n}^{\circ} 28$, septembre 1997 , pp. 76-93.

(5) Pour la première fois, lors du Ve congrès pénitentiaire international de Paris, en 1895, une section traite spécifiquement "des questions relatives à l'enfance et tions concernant les enfants martyrs. La loi s'attaque donc aux familles mauvaises, aux parents indignes, en aggravant les peines les concernant et en rendant plus aisée l'application de la déchéance de la puissance paternelle. De plus, si elle ne dépénalise pas directement la délinquance juvénile, elle offre la possibilité au juge de placer les jeunes auteurs de crimes et délits dans des institutions dépourvues de tout caractère pénal.

Cette dernière réforme s'inscrit dans un mouvement entamé depuis une quinzaine d'années. En effet, faute d'investissements financiers suffisants de l'Etat, l'amélioration des établissements anciens, souvent de vieilles colonies agricoles, privées ou publiques, directement issues ou inspirées par la loi d'août 1850, s'avère impossible. Sans abandonner totalement cette partie de leur programme - nombre de plans plus ou moins ambitieux en témoignent - les réformateurs recherchent des alternatives à l'enfermement institutionnel des jeunes. Ainsi, ils favorisent la renaissance et l'essor de sociétés de patronage, qui interviendraient non plus après mais avant, voire remplaceraient l'accomplissement par le jeune d'une partie de sa peine dans une colonie. ${ }^{(1)}$ Ces sociétés privées peuvent également s'occuper d'enfants en danger. Ils réfléchissent aussi aux possibilités d'utiliser les services des enfants assistés de l'Assistance publique pour s'occuper de ces mêmes catégories de population : jeunes délinquants primaires ou peu dangereux, enfants moralement abandonnés ou martyrs. La loi de 1889 explorait ces solutions; celle d'avril 1898 multiplie leur recours.

Notre contribution à l'analyse de la loi d'avril 1898 ne s'intéressera pas à l'économie générale de la loi, aux principes philosophiques, juridiques et éducatifs sur lesquels elle repose, aux visions de l'enfance, souvent bien contradictoires d'ailleurs, que l'on peut être tenté d'y lire, mais plus simplement aux établissements et aux institutions, publiques et privées, qu'elle mobilise pour en permettre l'exécution. La loi de 1898 possède donc une double nature, répressive envers les parents violents, abusifs, exploiteurs de leurs enfants, protectrice envers les jeunes victimes et coupables. C'est uniquement ce second aspect du texte qui nous intéresse, et en particulier ses articles 4 et 5 . Pour les enfants victimes, la loi de 1898 complète la loi de 1889. Elle en permet une 
meilleure application, en substituant à la déchéance totale de la puissance paternelle la déchéance limitée du droit de garde. Elle autorise également le juge d'instruction à prendre une mesure immédiate en ce qui concerne l'enfant. Pour les jeunes coupables, la nouvelle loi comprend une modification, discrète mais essentielle, de l'article 66 du code pénal. Elle offre au juge une alternative nouvelle, le sortant du dilemme consistant à remettre le jeune à une famille souvent tenue pour responsable de sa chute, à l'emprisonner pour de courtes et inutiles peines, ou encore à l'envoyer dans une maison de correction, souvent considérée comme néfaste à son avenir. Le juge peut dorénavant confier le jeune à une personne ou à une institution charitable, ainsi qu'à l'Assistance publique. La loi modifie donc de façon radicale les principes de l'éducation correctionnelle contenus dans le code pénal et précisés du point de vue des établissements par la loi de 1850. La nouvelle loi montre une certaine défiance envers les parents, ce qui n'est plus, à la fin du siècle, une nouveauté, mais aussi envers les colonies privées et publiques chargées de l'éducation correctionnelle des détenus; les colonies agricoles et pénitentiaires sont directement visées. À l'inverse, la loi met en avant de nouveaux groupements, les sociétés de patronage, ainsi que l'Assistance publique. Elle fait donc appel à de nouvelles institutions et en utilise une ancienne dans de nouvelles fonctions.

Les conséquences administratives et donc politiques de ce choix sont importantes. Très simplement, les parlementaires, éclairés et influencés par les sociétés de protection de l'enfance, décident de retirer des enfants destinés à l'Administration pénitentiaire pour les confier principalement à des sociétés privées et secondairement à des organismes départementaux. L'Etat perd donc une partie d'une population à éduquer, qui sera remise à des organismes relevant de l'initiative privée, du monde de la charité et de la bienfaisance. Or les explications budgétaires, bien que non négligeables, paraissent insuffisantes pour comprendre ce transfert de population. Il ne s'agit pas simplement d'alléger les dépenses du ministère de l'Intérieur, mais aussi d'un choix éducatif, qui reflète en même temps le climat des relations entre l'Etat et le monde de la protection de l'enfance : la primauté accordée par le légis- aux mineurs". Bulletin de

l'union des sociétés de

patronage, 1895 , pp. 106-

113. Un congrès international de protection de

l'enfance se tient à

Genève en 1896 :

“Comité de défense des enfants traduits en justice de Paris, séance du

2 décembre 1896",

Bulletin de l'Union des

sociétés de patronage,

1896, pp. 333-342.

(6) Nous employons ce mot, tel que le faisaient les hommes de la fin du XIXe siècle, dans son acception la plus large et la plus consensuelle, sans tenir compte des sensibilités religieuses et politiques. Les philanthropes de la fin du siècle ne répondent plus sur ces points particuliers à la même définition, si bien donnée par Catherine Duprat, que ceux du siècle précédent. Catherine DUPRAT, "Pour l'amour de l'bumanité" : le temps des philanthropes. La philanthropie 
parisienne des Lumières à la Monarchie de Juillet, tome 1, Paris, CTHS, 1993, XXXIV-485 p.

(7) Sur les congrès internationaux, Sylvette DUPONT, "Le mouvement international de protection de l'enfance, 1880-1914”, in Jeroen DEKKER, Sylvette DUPONT, Jacques-Guy PETIT et al., Enfants corrigés, enfants protégés. Genèse de la protection de l'enfance en Belgique, en France, aux Pays-Bas et au Québec (1820-1914), Angers, Louvain, Groningen, Montréal, Rapport de recherche au ministère de la justice français, 1995, pp. 252-277.

(8) Bernard SCHNAPPER, "La correction paternelle et le mouvement des idées au XIXe siècle”, Revue historique, avril-juin, 1980, pp. 320-349.

(9) "Autour du centenaire de la loi du 24 juillet 1889 relative à la protection des enfants lateur à l'initiative privée montre la confiance qu'il lui accorde. Ce transfert de population n'est possible que parce que le pouvoir législatif souhaite trouver une collaboration et même une coordination entre les services de l'Etat et les sociétés de charité privées. Cependant, la loi, en modifiant des habitudes, risque de provoquer certains mécontentements administratifs. Le pouvoir exécutif (ici les administrations) et le pouvoir parlementaire n'épousent pas forcément la même vision des choses.

Pour affiner notre compréhension du rôle des institutions dans la loi de 1898, nous allons examiner chacune d'entre elles, en commençant par celle qui se retrouve écartée, l'Administration pénitentiaire, puis en poursuivant par celles qui semblent bénéficier de la loi, les sociétés de patronage et enfin l'Assistance publique. Nous nous situerons principalement à deux moments, lors des débats parlementaires, puis durant les premières années d'exécution de la loi, alors qu'une nouvelle jurisprudence se construit, imposant des ajustements entre ces différentes institutions. ${ }^{(11)}$

\section{LA DÉFIANCE À L'ÉGARD DE L'ADMINISTRATION PÉNITENTIAIRE ET DES MAISONS DE CORRECTION}

La nouvelle loi offre donc au magistrat devant traiter le cas d'un jeune délinquant une alternative à la remise aux parents ou à la "flétrissure" de l'envoi en correction. L'Administration pénitentiaire et les colonies publiques et privées se retrouvent, si ce n'est éliminées, du moins écartées de fait. Le juge peut continuer à leur adresser un jeune, mais il peut aussi faire le choix temporaire ou définitif de le confier à une société charitable ou à l'Assistance publique. Il n'y est pas contraint, mais il en a la possibilité. De plus, le délai entre la mesure temporaire et la mesure définitive lui permet de mettre en place une sorte d'observation de l'enfant. Les quelques semaines dont il dispose alors l'autorisent à se rendre compte de l'état de moralité et des capacités d'amendement du jeune détenu. Car la remise à une société de patronage ou à l'Assistance publique ne doit pas être générale, mais se limiter aux jeunes qui ne sont pas définitivement "perdus"; la correction demeure pour tous ceux qui paraissent inamendables. 
Ces nouvelles possibilités offertes au juge, même si les partisans de la loi infirment cette idée, sont largement inspirées par la critique récurrente des institutions correctionnelles qui se développe depuis les années 1870. La commission parlementaire de 1872 a mis en lumière les dysfonctionnements des colonies privées : violence, sous-effectif du personnel, absence de véritable formation professionnelle des jeunes, insuffisance de l'instruction, absurdité du choix du travail agricole pour des détenus essentiellement issus des milieux urbains, etc. ${ }^{(12)}$ Les colonies publiques aux effectifs pléthoriques n'apparaissent ni mieux organisées, ni plus vivables pour les colons. Les difficultés économiques de la fin du siècle aggravent la situation, fragilisant hommes et institutions. Les établissements ne trouvent pas l'équilibre budgétaire indispensable à leur survie. Ouvertures et fermetures se succèdent à un rythme alors extrêmement rapide; la géographie des institutions correctionnelles est en perpétuel bouleversement. S'il existe, en 1880, quatre établissements publics pour cinquante-six établissements privés, il ne se trouve plus, en 1912, que dix établissements privés et treize établissements publics. ${ }^{(13)}$ Dans le domaine précis de l'éducation correctionnelle, l'Etat peu à peu se substitue donc à l'initiative privée défaillante et découragée; globalement, cette évolution satisfait l'Administration pénitentiaire, mais elle l'affaiblit aussi.

Surtout que, depuis trente ans, les plans de réforme des établissements correctionnels se multiplient, sans effets. Les revues spécialisées en comportent toutes plusieurs. ${ }^{(14)}$ Mais l'argent fait toujours défaut. Les idées de transformation plus ou moins radicale de ces maisons de correction prennent naissance dans toutes les instances de réflexion étatiques ou para-étatiques, mais les budgets de l'Administration pénitentiaire ne permettent pas leur réalisation. Quelques innovations se mettent en place, comme la création des "écoles de réforme", mais elles apparaissent très insuffisantes. ${ }^{(15)}$ Reposant sur leur incapacité à se transformer, la critique des colonies agricoles se répand. L'opprobre qui pèse sur elles passe du cercle étroit des spécialistes à un public plus large. D'autant que, dans les années 1880, l'affrontement entre le pouvoir républicain et l'Eglise catholique a permis que soient révélés dans la presse nationale certains scandales de mauvais traitements à enfants. maltraités et moralement abandonnés ", Archives aquitaines de recherche sociale, $\mathrm{n}^{\circ}$ spécial 19891990, 168 p.

(10) Sur le premier modèle de Société de patronage, Catherine DUPRAT, Usage et pratiques de la philanthropie. Pauvreté, action sociale et lien social à Paris, au cours du premier XIXe siècle, Paris, Comité d'histoire de la sécurité sociale, 1997, volume 2, pp. 751-753.

(11) Outre les débats parlementaires du Sénat et de la Chambre des députés, nos sources seront principalement constituées des procèsverbaux des séances de la Société générale de prisons, du Comité de défense des enfants traduits en justice de Paris et de l'Union nationale des sociétés de patronage. On trouve les premiers dans la Revue pénitentiaire et les autres dans le Bulletin de l'Union des sociétés de patronage. 
(12) Voir la déposition du directeur de l'Administration pénitentiaire lors de la séance du 1872, le rapport de Félix Voisin et surtout le rapport resté confidentiel de Victor Bournat. Respectivement : Enquête parlementaire sur le régime des établissements pénitentiaires, Paris, 1874, volumes 1 et 6 . Victor BOURNAT, Rapport présenté à la commission d'enquête parlementaire sur le régime des établissements pénitentiaires sur les questions suivantes : Doit-on préférer pour l'éducation correctionnelle le système des colonies privées ou celui des colonies publiques? Doit-on maintenir sur ce point les dispositions de la loi du 5 août 1850 ?, Paris, Imprimerie nationale, 1875, 31 p.

(13) Le secteur public se compose de huit colonies pénitentiaires, deux colonies correc-
Plus récemment, dans Le Coupable, François Coppée a ému avec son jeune héros jeté dans une colonie corruptrice. ${ }^{(1)}$ En conséquence, les juges hésitent de plus en plus à placer les jeunes dans ces établissements. ${ }^{(17)}$

En fait, la nouvelle réforme de l'article 66 contenue dans la loi de 1898 leur offre la possibilité de développer une pratique qu'ils avaient testée lors de la décennie précédente. En contradiction avec la loi d'alors, des juges plaçaient déjà des enfants auprès des sociétés de patronage. Mais la Cour de cassation, en plusieurs occasions, avait dénoncé ces nouvelles mesures, en annulant même plusieurs. ${ }^{(18)}$ Désormais, les juges bénéficient légalement de cette nouvelle possibilité. Un jeune détenu amendable peut être confié à une institution charitable.

Ce nouveau choix n'est pas sans conséquence pour l'Administration pénitentiaire et pour les colonies, qui se voient finalement remettre les enfants les plus difficiles. S'amorce ainsi une évolution, qui se développera ensuite et que confirme l'augmentation du nombre des établissements publics, rejetant sur les colonies de l'Etat les enfants qui ont été condamnés et ceux qui, bien qu'acquittés, paraissent les plus "inamendables". On trouve ainsi une répartition souhaitée par de nombreux agents, dont certains inspecteurs des prisons, entre l'Etat, qui s'occupe de répression, et les établissements relevant de l'initiative privée, qui éduquent les enfants assistés et les délinquants les moins dangereux. Par ce côté, la loi pouvait séduire les fonctionnaires de l'Administration pénitentiaire, ${ }^{(19)}$ même si elle marquait très nettement une défiance à l'égard des établissements dont ils avaient la responsabilité ou le contrôle.

\section{LA FLORAISON DES SOCIÉTÉS DE PATRONAGE}

À l'inverse, les sociétés de patronage apparaissent comme les grandes bénéficiaires de la loi. Le texte semble même avoir été conçu en raison de leur existence. La proposition initiale défendue par René Bérenger prévoyait même de leur confier le droit de poursuivre les attentats commis sur les enfants, soit par voie de citation directe, soit en se portant partie civile. ${ }^{(20)}$ Par là, le sénateur inamovible pensait pouvoir multiplier à la fois 
les actions de prévention (les présidents d'associations pouvant intervenir facilement auprès des parents lors de faits peu graves), mais aussi les actions répressives (ces mêmes présidents n'hésitant pas à signaler les affaires auprès des tribunaux). Les parlementaires ont renoncé à cette mesure qui, pensaient-ils, pouvait représenter un acte de défiance à l'égard du parquet et qui surtout rentrait en opposition avec un principe du droit français : un particulier ne peut poursuivre la répression d'un délit que s'il y a un intérêt direct et personnel et qu'autant qu'il a été lésé par le dit délit. En France, un individu ne peut, à la différence de l'Angleterre et de l'Allemagne, poursuivre une action en justice dans un but d'intérêt social. ${ }^{(21)}$

Ecartées sur ce point, les associations protectrices conservent cependant une position dominante dans l'accueil des enfants. Il paraît évident que peu de jeunes seront confiés directement à des personnes charitables, faute de garanties suffisantes, mais qu'à l'inverse beaucoup le seront aux sociétés de patronage. En multipliant par rapport à la loi de 1889 le nombre d'enfants qui leur seront potentiellement remis, les parlementaires de 1898 prennent en compte le développement de ces sociétés et la structuration de leur mouvement. Progressivement elles s'implantent sur l'ensemble du territoire national; peu de départements n'en abritent pas au moins une. Les premiers congrès nationaux de patronage tiennent une comptabilité précise de ces implantations et des créations. Une enquête, entamée en 1893, en comptabilise 106 en France et aux colonies. ${ }^{(22)}$ Depuis 1894, à l'instigation d'Emile Cheysson, existe le Bureau central de l'Union des sociétés de patronage, une instance d'information et d'entraide. ${ }^{(23)}$ À partir de 1895, le Bureau publie une revue régulière.

Peu à peu, ces associations sortent de leur mission première, le patronage des libérés (une action intervenant après l'incarcération ou l'envoi en correction), pour s'intéresser à l'éducation des enfants de justice. ${ }^{(24)}$ Elles paraissent les mieux armées pour trouver un véritable patron attentif à l'intérêt des jeunes et bienveillant. La loi de 1889 leur avait déjà confié un rôle important, mais cette fois elles apparaissent comme la principale solution aux problèmes de l'enfance victime et de l'enfance coupable. Le législateur les a inscrites au centre de son dispositif; il leur a donné une condition privilégiée. Il paraît aller de soi, et les premières tionnelles, ainsi que de trois établissements pour filles. De plus, deux prisons reçoivent les enfants placés en détention préventive : La Petite Roquette et Fresnes. Henri GAILLAC, Les Maisons de correction, Paris, Cujas, 1991 (2e édition), p. 261.

(14) Par exemple : Louis PUIBARAUD, "Les Maisons d'éducation préventive et correctionnelle. Essai d'un plan de réforme de la loi du 5 août 1850 sur les jeunes détenus", Paris, Gazette du Palais, 1894, 63 p.

(15) David NIGET, L'Enfant redressé. La colonie agricole pénitentiaire de Saint-Hilaire (18601890), Université d'Angers, mémoire de maîtrise, 1994, p. 36.

(16) François COPPEE, Le Coupable, Paris, Lemerre, 1897, 327 p.

(17) Il s'agit d'un thème récurrent du discours des réformateurs. 
(18) Arrêté du 26 juillet 1893, par exemple.

(19) C'était une revendication de Charles Lucas. Charles LUCAS, Observations présentées à la Société générale des prisons sur la révision de la loi du 5 août 1850 , relative aux colonies agricoles pénitentiaires des jeunes détenus, Paris, A. Chaix, 1879, 12 p. Charles LUCAS, Observations sur la colonisation agricole pénitentiaire, supplémentaires à celles présentées à la séance $d u$ 3 mars 1879 de la Société générale des prisons, Paris, A. Chaix, 1879, 12 p.

(20) Mesure controversée, mais réclamée dans de nombreux ouvrages et brochures, en particulier Paul NOURISSON, Des Réformes à apporter au Code pénal pour fortifier la répression des délits et des crimes contre la moralité des mineurs de seize ans, et de la participation des sociétés privées à la poursuite de ces faits, Paris, au bureau du journal $\mathrm{La}$ décisions le confirmeront, que les juges s'adresseront prioritairement aux sociétés de protection. Leur position parait d'autant plus facilitée qu'elles n'ont plus besoin d'un agrément spécifique, ${ }^{(25)}$ qu'aucun contrôle n'est prévu sur leur action ${ }^{(26)}$ et qu'elles peuvent d'ailleurs refuser d'accueillir un jeune. ${ }^{(27)}$ Avant toute mesure, il doit exister une concertation préalable entre les juges et les sociétés, fonction qu'assument dans plusieurs tribunaux les Comités de défense des enfants traduits en justice.

La primauté accordée aux sociétés de patronage montre l'attachement des parlementaires à l'initiative privée dans les domaines de la justice des jeunes délinquants et de l'assistance aux mineurs en danger. En une période de bonne coordination, bien que non dénuée de conflits d'ajustement, entre les instances ministérielles de l'Assistance publique et les organismes de la charité privée, à la suite du ralliement des catholiques à la République et de l'Encyclique Rerum novarum, le parlement modéré entérine donc le développement de l'initiative privée. Il semble y voir une solution pour les jeunes victimes et coupables. Mais les conditions offertes aux sociétés privées, en particulier les absences de contrôle par des inspecteurs et d'obligation d'assistance, dépassent même les normes qui se mettent alors en place dans les autres secteurs de l'assistance et de la bienfaisance. ${ }^{(28)}$ Jamais l'initiative privée n'a autant été favorisée par rapport aux administrations publiques.

Il existe cependant un problème de taille : le financement de ces sociétés. En effet, le législateur n'a rien prévu pour contribuer à leur intervention. Aucune ligne budgétaire ministérielle ne semble même pouvoir accueillir les subventions possibles. Pendant plusieurs années, les patronages travaillent donc presque sans argent public, mais cela entrave bien sûr leur capacité d'action. La lecture des débats donne l'impression que les parlementaires, bousculés par le calendrier des élections législatives, se sont empressés de voter une loi entérinant le rôle de l'initiative privée et ont remis à plus tard la question de son financement. Ils voulaient assurer définitivement la primauté de l'action associative. Mais le ministère se montrera ensuite avare de ses aides. En 1897, l'inspecteur général des prisons Granier, devant l'assemblée générale de l'Union des sociétés de patronages, avait déclaré que l'Etat n'est ni "indifférent" ni "hostile" sur cette question du patronage, mais "neutre". 
Il promettait aux ouvres le concours des fonctionnaires et des agents de l'Administration pénitentiaire, mais aucune nouvelle aide financière. ${ }^{(30)}$

\section{LASSISTANCE PUBLIQUE, MALGRÉ ELLE}

Si l'on regarde la place accordée à l'Assistance publique dans la loi, on s'aperçoit que les articles 4 et 5 la mentionnent en dernier recours dans l'énumération des solutions offertes au juge; cette situation n'est pas fortuite. Les rédacteurs de la loi ont ainsi voulu montrer que l'Assistance publique ne doit intervenir qu'en dernière possibilité, si le juge n'a pas trouvé une autre solution dans le monde charitable. C'est seulement en l'absence de sociétés de patronage ou en cas de refus de leur part de s'occuper d'un jeune que l'Assistance peut se voir confier un enfant. Des articles publiés dans la presse nationale ${ }^{(31)}$ comme dans les revues juridiques confirment cette interprétation. ${ }^{(32)}$ Le recours à une Assistance publique mal équipée pour s'occuper de ces enfants apparaît être une solution ultime. L'Assistance présente cependant, et cela explique son inscription dans la loi, des garanties de permanence et de pérennité que ne possèdent pas les institutions privées. Elle existe dans tous les départements, elle dispose de quelques équipements, d'un réseau de familles d'accueil et, surtout, elle ne risque pas de fermer ses portes. En somme, le recours à l'Assistance publique apparaît meilleur que celui à l'Administration pénitentiaire, mais bien inférieur au recours aux sociétés charitables; son intervention doit rester subsidiaire.

Cette loi provoque au sein de cette Administration une véritable révolte, qui a déjà été analysée ${ }^{(33)}$ et sur laquelle je m’étendrai donc peu. Très vite, dans différents départements, les services concernés refusent d'assurer l'accueil des jeunes. Dans la Seine, l'Assistance publique met d'abord en œuvre, au sein de l'hospice Denfert-Rochereau, un système d'observation visant à séparer ceux "susceptibles d'un amendement relativement facile " et "ceux d'une nature vicieuse ou indisciplinée ", qui doivent retourner en maison de correction. Puis elle abandonne cette pratique, se contentant de refuser la très grande majorité des enfants, même parmi
Loi, 1897. Mais pour

l’occasion René Bérenger est directement inspiré par H. Joly : “Assemblée générale de l'Union sociétés de patronage du 15 juin 1897”, Bulletin de l'Union des sociétés de patronage, 1897, pp. 116117. C'est également Bérenger qui introduit les associations au centre du dispositif de garde des enfants.

(21) Une erreur commune à l'époque (visible en particulier dans plusieurs débats parlementaires sur des sujets variés) attribue cette faculté à la seule Société protectrice des animaux; il n'en est évidemment rien. Sur ce point particulier : Eric PIERRE,

Amour des hommes, amour des bêtes. Discours et pratiques protectrices dans la France du XIXe siècle, Doctorat d'histoire, Université d'Angers, 1998.

(22) Enquête sur le patronage, sous la direction du Bureau central de 
l'Union des sociétés de patronage de France, Bordeaux, impr. de J. Durand, 1896. Et Congrès national du patronage des libérés, Bordeaux, 1896.

(23) Emile CHEYSSON, Le Bureau central de l'Union des sociétés de patronage. Congrès national de patronage des libérés, Lyon, (20-22 juin 1894), Lyon, Storck, 1894, 22 p.

(24) Les monographies sur les patronages commencent à se multiplier. Parmi les plus complètes et intéressantes : Dominique DESSERTINE, La Société lyonnaise pour le sauvetage de l'enfance (1890-1960). Face à l'enfance en danger, un siècle d'expérience de l'internat et du placement familial, Toulouse, Erès, 1990, 218 p.

(25) À la différence de la loi de 1889 .

(26) L'absence de contrôle est justifiée par la crainte de décourager les initiatives privées : "Bureau central du les plus jeunes, âgés de 8 à 10 ans. ${ }^{(34)}$ En fait, rares sont les départements qui acceptent sans protester cette nouvelle population. ${ }^{(35)}$

Parallèlement, plusieurs responsables de l'Assistance dénoncent, d'abord discrètement, puis avec plus de vigueur, les nouvelles populations qu'on leur impose. Ils mettent en avant leur inaptitude à gérer ces jeunes, de "véritables petits criminels", incapables de rentrer dans le cadre $\mathrm{du}$ traditionnel placement familial. Ils craignent que ces "enfants vicieux" ne contaminent les «enfants bonnêtes » recueillis par l'Assistance publique. ${ }^{(3)}$ Ils regrettent que l'on ait substitué à l'éducation correctionnelle de l'enfance délinquante et difficile "l'éducation hospitalière ». ${ }^{(37)}$ Pour Loys Bruèyre, "la loi est inapplicable ", car l'Assistance publique, surtout en province, n'est pas organisée pour recevoir ces jeunes. ${ }^{(38)}$ À sa suite, toute la hiérarchie se mobilise pour intervenir à différents niveaux : ministère de l'Intérieur, Parlement, Conseil supérieur de l'Assistance publique, etc. Des fonctionnaires sortent de leur devoir de réserve pour faire des articles critiques dans la presse spécialisée ou même généraliste. Par exemple, dans un rapport sur les questions d'assistance daté de 1903, E. Ogier, chef de service à l'inspection générale, met en lumière les difficultés causées par les enfants des lois de 1889 et 1898 . Pour lui, ils possèdent nombre de tares physiques et psychiques, héréditaires et acquises. ${ }^{(39)} \mathrm{Il}$ dénonce surtout l'obligation faite à l'Assistance publique de les recevoir, alors que les sociétés de patronages décident des enfants dont elles se chargent. Il souhaite la remise de ces derniers à l'Etat. Il conclut enfin que la loi de 1898 doit être abrogée, car elle comporte «lacunes, contradictions, difficultés d'application, inégalités possibles de traitement".

Ces discours et leur répétition portent. Malgré les protestations des juristes, qui estiment que l'Assistance ne peut pas « se dérober à une obligation de garde » et à une mesure prise par un tribunal, ses responsables obtiennent l'élaboration de dispositifs administratifs puis législatifs leur permettant de rejeter en partie la nouvelle population des jeunes. La loi de 1904 a, entre autres, cet objectif ${ }^{(4)}$; la loi de 1912 sur les tribunaux pour enfants réserve l'intervention de l'Assistance publique aux plus jeunes. ${ }^{(4)}$ Faute de volonté politique, peu de départements prennent les engagements financiers nécessaires à l'ouverture des écoles professionnelles prévues pour accueillir les 
pupilles difficiles. ${ }^{(42)}$ Bien au contraire, certains conseils généraux passent des accords avec des établissements privés, qui ne sont autres, pour certains, que d'anciennes colonies pénitentiaires, pour y placer des enfants : on retrouve parmi eux le Luc, Bologne et Mettray. ${ }^{(43)}$ En définitive, des jeunes qui avaient été confiés à l'Assistance publique pour leur épargner l'envoi en correction se retrouvent par ce détour dans des colonies. ${ }^{(44)}$

L'administration détournait ainsi l'esprit de la loi, contribuant à dévaloriser une justice des mineurs déjà bien critiquée.

Pour conclure, il faut remarquer en premier lieu l'impréparation de la loi, visible dans les nombreuses hésitations des tribunaux qui ont suivi sa promulgation. ${ }^{(4)}$ Si ses orientations générales ont déjà été largement débattues dans les cercles des spécialistes, la rédaction précise des articles n'a pas fait l'objet de concertation. L'aspect réactif du législateur face à des événements tragiques a déjà été noté. ${ }^{(4)}$ La loi est préparée rapidement, votée exceptionnellement vite, alors que la parité des chambres tend habituellement à allonger les processus de production législatifs. De plus, le texte définitif amalgame des propositions venues de plusieurs parlementaires et comporte des aspects très différents. Il forme une synthèse mal équilibrée, ou plutôt un catalogue de mesures variées. La lecture des revues juridiques confirme cette impréparation. On ne trouve presque aucune discussion préalable au sein de la Société générale des prisons, du Comité de défense des enfants traduits en justice, ${ }^{(47)}$ ou encore de l'Union des sociétés de patronage. Sans doute la réforme est-elle dans "l'air du temps"; elle provient des critiques portées aux insuffisances de la loi de 1889 et aux établissements correctionnels anciens. Le 9 février 1898, le sénateur Bérenger vient présenter une partie de son programme au Comité de défense des enfants traduits en justice de Paris, celle permettant aux sociétés de se porter partie civile, alors que les débats parlementaires sont déjà largement avancés. Le même Comité discute encore, dans sa séance du 4 mai 1898, alors que la loi a déjà été adoptée, un rapport de Paul Nourisson sur les réformes à apporter au code pénal pour fortifier la répression des crimes et délits contre la moralité des mineurs de 16 ans ${ }^{(48)}$ : étrange décalage entre une loi déjà
19 janvier 1904”, Revue

pénitentiaire, 1904, p. 266.

(27) C'est le principal

débat des premiers mois d'application de la loi.

La question sera tran-

chée en faveur des sociétés de patronage. Dès décembre 1898,

E. Passez, avocat à la

Cour de cassation, estime que les sociétés n'ont aucune obligation à recevoir des enfants. Il faut, selon lui, qu'elles aient des représentants accrédités auprès des tribunaux et qu'une entente se dégage entre ceuxci et les juges : "Comité de défense des enfants traduits en justice, assemblée générale du 17 décembre 1898", Bulletin de l'Union des sociétés de patronage, 1898, p. 411. De nombreuses gravures $\mathrm{du}$ début du XXe siècle montrent les membres des patronages sélectionnant les jeunes lors des audiences des tribunaux.

(28) Sur ces points, 
voir l'article de Colette BEC, "Deux congrès internationaux d'assistance (Paris 1889-1900). Temps forts des rapports public-privé”, in Colette BEC, Catherine DUPRAT, Jean-Noël LUC, Jacques-Guy PETIT, Philanthropies et politiques sociales en Europe (XVIIIe$X X e$ siècles), Paris, Anthropos, 1990, pp.145-157.

(29) En 1896, la subvention aux sociétés de patronage s'élève à 140.000 francs; en 1897, elle est d'abord rabaissée à 120.000 francs, puis rétablie dans sa quasi intégralité. La subvention ne connait pas d'augmentation notable ensuite, malgré le vote de la loi de 1898 : "Note sur le crédit de subvention aux institutions de patronage des prisonniers libérés", Bulletin de l'Union des sociétés de patronage, 1896, pp. 377-381.

(30) L'inspecteur général des prisons Granier délibérée et des débats portant sur le même sujet. Cette précipitation explique que, comme en 1889, les parlementaires n'ont pas prévu d'augmentation des crédits pour le traitement des populations nouvelles. Les sociétés privées doivent patronner des jeunes sans que des subventions spécifiques soient dégagées. Elles réclameront, dès le début du XXe siècle, des aides, seule solution pour que leur action ait l'envergure souhaitée par le législateur. L'Assistance publique également se voit confier une extension de sa mission, sans argent supplémentaire et sans qu'elle possède le personnel et l'équipement adéquats. Cela entraîne une protestation nourrie de plusieurs de ses responsables et un refus obstiné de certains services de s'ouvrir aux nouvelles populations.

L'impéritie parlementaire s'explique en partie par la crise que traversent alors la société française en général et la justice des mineurs en particulier. Les statistiques officielles et, surtout, leurs interprétations montrent une montée de la délinquance juvénile, la précocité des jeunes criminels et l'importance de la récidive. Les criminologues insistent sur les maux sociaux, dans un discours qui s'inscrit dans une crainte générale de la dégénérescence. ${ }^{(4)} \mathrm{La}$ presse, friande de faits divers, met en avant quelques affaires monstrueuses. La société française, comme l'a montré Michelle Perrot, prend peur de la jeunesse. ${ }^{(50)}$ Dans ce contexte, il faut agir. Et l'adoption d'une loi semble bien être la chose la plus facile. Ainsi, les années 1890 et 1900 montrent une intense activité législative dans le domaine de l'enfance de justice qui, et les contradictions entre les textes des lois le prouvent, ne répond pas à une véritable politique de fond. Chaque texte semble apporter une solution nouvelle, la déchéance de la puissance paternelle en 1889 , le patronage et "l'éducation hospitalière" en 1898, l'élévation de l'âge de la majorité pénale en 1906, le tribunal pour enfants et la liberté surveillée en 1912, etc., mais à chaque fois il manque une condition indispensable à la réussite de l'entreprise : l'attribution des budgets nécessaires au bon fonctionnement des réformes.

Notons ensuite que la loi modifie les équilibres entre les institutions, sans avoir ni organisé ni évalué l'importance des transferts de population. Très vite, dans les tribunaux, les juges utilisent massivement des sociétés de patronage dépassées par l'ampleur de la tâche et une 
Assistance publique réticente, tout en poursuivant le retrait des jeunes des établissements correctionnels. La loi de 1904 autorise l'Assistance publique a renvoyer vers les colonies privées et publiques ses "jeunes difficiles et vicieux». Mais avant ce premier correctif, négocié entre les administrations, ${ }^{(51)}$ les colonies se vident. Ce mouvement, souhaité par les réformateurs et les philanthropes, finit par dépasser leurs espérances et même par les préoccuper. En 1903, le Bureau central de l'Union des sociétés de patronage décide d'intervenir pour changer le cours des choses. Une pétition adressée aux membres de la magistrature, et signée " de tous les présidents d'auvres et de tous les criminalistes les plus imminents" présente les avantages de l'envoi en correction. Les signataires regrettent à la fois la remise des enfants à la famille, les courtes peines de prison et les placements inopportuns en patronage. "Et puis, écrivent-ils, la maison de correction n'est pas, comme on se l'imagine trop facilement, une sorte de bagne pour enfants. Les maisons de correction, les colonies pénitentiaires modernes ressemblent à des écoles; c'est bien à tort qu'on leur fait une mauvaise réputation. " ${ }^{(52)}$

Ces réformateurs, dont plusieurs appartiennent à des conseils d'administration de colonies privées, s'inquiètent soudainement pour la survie des établissements dont ils ont la charge. Ils craignent également l'engorgement des sociétés de patronage et leur investissement par des jeunes trop peu amendables. Ils proposent enfin, dans la pétition, de mettre en place un système combinant envoi en correction et patronage : après quelques jours de prison préventive, le jeune serait placé par le juge en correction, mais au lieu de le faire transférer dans une colonie ce dernier le confierait à une société de patronage. Ainsi la menace de l'envoi en correction continuerait à peser sur sa tête et le guetterait à la moindre faute. Les réformateurs proposent donc, dans une pétition, de détourner ouvertement la loi. Ils demandent au juge de prononcer une mesure et d'en faire appliquer une autre. C'est à la fois le signe de l'inefficacité de la loi et des libertés que l'on peut prendre avec, dès lors qu'il s'agit de mineurs. Cependant, de tels usages contribuent à affaiblir encore un peu plus une justice déjà traversée par des crises récurrentes, même s'ils sont conçus avec les meilleures intentions du monde, et bien évidemment dans l'intérêt de l'enfant. déclare aussi que "les questions d'argent sont odieuses ", et il ajoute "j'en parlerai d'autant moins que je les considère subsidiaires dans une cuvre spéciale d'assistance ". Il estime enfin " que l'appui moral que peut offrir l'administration " est beaucoup plus important. "Assemblée générale du 15 juin 1897', Bulletin de l'Union des sociétés de patronage, 1897, pp. 101-111.

(31) En particulier par H. Rollet.

(32) Plusieurs juges rédigent même des mesures confiant prioritairement les enfants aux sociétés privées et, en cas de défaut, à

l'Assistance publique.

E. Passez défend cet usage : Emile PASSEZ, "Loi du 19 avril 1898.

Dispositions nouvelles contenues dans les articles 4 et 5 à l'égard des enfants coupables de crimes et de délits", Bulletin de l'Union des 
sociétés de patronage, 1898, pp. 350-359.

(33) Voir Pascale LEFEBVRE, "La Tentation de l'exclusion : l'Assistance publique et les enfants difficiles ou vicieux, fin XIXe-début XXe siècles”, in Adré GUESLIN, Dominique KALIFA, Les Esclaves en Europe, 1830-1930, Paris, l'Atelier, pp. 202-214.

(34) Le juge Albanel dénonce cette pratique devant le Comité de défense des enfants traduits en justice de Paris.

(35) "Séance du 6 juillet 1898”, Bulletin de l'Union des sociétés de patronage, 1898, pp. 325326. Une enquête de 1899 indique que seulement 40 départements ont reçu des enfants dans leurs services d'assistance. La plupart sont conservés dans des hospices et ne bénéficient pas de placement en famille d'accueil.

“Comité de défense des enfants traduits en justi- ce de Paris, séance du

14 juin 1899”, Bulletin de

l'Union des sociétés de

patronage, 1899, pp. 157.

(36) Déclaration de

Loys Bruèyre devant le

Comité de défense des enfants traduits en justice de Paris : "Séance du 3 mai 1899", Bulletin de l'Union des sociétés de patronage, 1899, pp. 155-156.

(37) Expressions empruntées à la thèse de droit d'un inspecteur départemental de l'Assistance publique : Julien LELIEVRE, L'Enfance difficile, vicieuse ou délinquante et

l'Assistance publique départementale, thèse de droit, Rennes, impr. Donneur et Ruech, 1921, 123 p.

(38) Il réclame aussi que l'Administration pénitentiaire paye pour les enfants dont la charge cesse de lui incomber avec la nouvelle loi. Ibid.

(39) Albert RIVIERE donne une présentation du rapport d'Emile OGIER dans la Revue pénitentiaire, 1903, pp.

1441-1447.

(40) Loi du 28 juin 1904 "relative à l'éducation des pupilles difficiles ou vicieux de l'Assistance publique”. Les pupilles vicieux seront remis à l'Administration pénitentiaire, alors que ceux qui ne sont que difficiles seront élevés dans "des établissements spéciaux d'assistance”, les “écoles professionnelles”.

(41) Eric PIERRE, "Juge de papier, juge de chair, du tribunal pour enfants et adolescents au juge des enfants", in Monique CHARVIN, Jean-François GAZEAU, Eric PIERRE, Françoise TETARD, Recherche sur les juges des enfants.

Approches historique, démographique, sociologique, Rapport de recherche au ministère de la justice, juillet 1996, pp. 9-63.

(42) À l'exception des départements de la Marne, de la Seine (établissement de
Montesson) et de la Seine-Inférieure (établissement d'Aumale).

(43) En principe, les enfants de l'Assistance publique ne devaient pas se retrouver avec les enfants de l'Administration pénitentiaire. Mais, à Mettray, par exemple, la direction se contente de créer une "famille" spéciale pour les plus jeunes. À partir de 13 ans, il n'existe plus de différence de traitement. Raoul LEGER, La Colonie agricole et pénitentiaire de Mettray, souvenirs d'un colon, Paris, L'Harmattan, 1997, 165 p.

(44) Il en est de même pour les "pupilles vicieux" renvoyés directement vers l'Administration pénitentiaire. Pascale LEFEBVRE,"La tentation de l'exclusion : l'Assistance publique et les enfants difficiles ou vicieux, fin XIXe-début XXe siècles” (à paraître).

(45) Des interprétations très contradictoires 
naissent en particulier au sujet du passage de la mesure provisoire à la mesure définitive. En cas de délit, pour éviter un véritable procès à des jeunes amendables, certains juristes proposent que le juge d'instruction se contente de conserver le dossier de l'enfant, prolongeant ainsi la mesure provisoire "aussi longtemps qu'il le croit utile", d'autres souhaitent qu'il prenne une ordonnance de non-lieu tout en maintenant le jeune dans un patronage. Les plus formalistes réclament un passage devant le tribunal permettant de transformer la mesure provisoire en mesure définitive.

"Comité de défense des enfants traduits en justice, séance du 19 juillet 1899”, Bulletin de l'Union des sociétés de patronage, 1899, p. 159. La pratique des tribunaux emprunte ces différentes voies.

(46) Voir en particulier les travaux de JeanJacques YVOREL.

(47) Paul Nourisson a cependant présenté un rapport au Comité de défense des enfants traduits en justice de Paris dans la séance du 28 juin 1897. Outre la brochure déjà mentionnée, voir Bulletin de l'Union des sociétés de patronage, 1897 , pp. 130-132. Le 9 février 1898, Bérenger s’appuie largement sur le travail de Paul Nourisson.

Bulletin de l'Union des sociétés de patronage, 1898, pp. 21-25.

(48) Bulletin de l'Union des sociétés de patronage, 1898, p. 179 et pp. 326329.

(49) Sur la criminologie, Laurent MUC-

CHIELLI (sous la direction de), Histoire de la criminologie française, Paris, L'Harmattan, 1995, 535 p.

(50) Michelle PER-

ROT, "Quand la société prend peur de sa jeunesse, au 19e siècle”, in Les
Jeunes et les autres. Contribution des sciences de l’homme à la question des jeunes, Vaucresson, Criv, 1986, pp. 19-27.

(51) Pascale LEFEBVRE, "La Tentation de l'exclusion : l'Assistance publique et les enfants difficiles ou vicieux, fin XIXe-début XXe siècles” (à paraître).

(52) "Circulaire du Bureau central”, Revue pénitentiaire, 1903, pp. 1401-1403.

(53) "La tâche des Sociétés de patronage est difficile, ardue, à l'égard des enfants qui souvent, trop souvent, n'ont jamais eu que de mauvais exemples sous les yeux. Avec ces enfants, il ne suffit pas de petites exhortations familières, de suite oubliées, de bons points donnés ou retirés; il faut quelque chose de plus : il faut que l'enfant sente bien que, s'il manque à sa promesse, s'il ne suit pas les conseils qui lui ont été donnés, il en sera puni immédiatement par la perte de sa liberté". Ibid., pp. 1405-1406. 